\title{
Tea polyphenols protect against ischemia/reperfusion-induced liver injury in mice through anti-oxidative and anti-apoptotic properties
}

\author{
JIN TAO ${ }^{1}$, XINHONG SHEN $^{1}$, YONGHONG $\mathrm{AI}^{2}$ and XIAOJING HAN ${ }^{1}$ \\ ${ }^{1}$ Department of Nephrology; ${ }^{2}$ Blood Purification Room, Xinxiang Central Hospital, Xinxiang, Henan 453000, P.R. China
}

Received May 25, 2015; Accepted January 19, 2016

DOI: $10.3892 / \mathrm{etm} .2016 .3789$

\begin{abstract}
Tea polyphenols (TPs), which are derived from tea extracts, are a class of chemicals containing polyphenol hydroxyls that have been observed to have strong anti-oxidative properties. Previous studies have demonstrated that TP can protect against hepatic ischemia/reperfusion (I/R) injury; however, the underlying mechanism remains unknown. In the present study, the mechanism underlying TPs protective effects against I/R-induced liver damage was investigated, focusing on its anti-oxidative and anti-apoptotic bioactivities. C57BL/6 mice were used to establish a hepatic I/R-induced injury model, and liver injury was analyzed using a biochemical assay. The results from the current study demonstrated that the serum expression levels of aspartate aminotransferase (AST) and alanine aminotransferase (ALT) were significantly increased in mice following hepatic I/R injury, while the ratio of hepatic glutathione (GSH)/oxidized GSH (GSSG) was reduced, indicating that liver damage had occurred. In mice that were orally administered with TP $(50 \mathrm{mg} / \mathrm{kg}) 1 \mathrm{~h}$ prior to I/R-induced injury, the extent of liver injury was significantly attenuated. It was also observed that I/R injury significantly decreased the mRNA and protein expression levels of cytokine-inducible nitric oxide synthase in liver tissues, and this was also attenuated by pretreatment with TP. Furthermore, pretreatment with TP significantly attenuated the I/R-induced increase in liver cell apoptosis, and the expression level and activity of pro-apoptotic proteins in the liver, indicating that I/R-induced liver cell apoptosis is inhibited by TP. In conclusion, the results in the present study suggest that TP protects against hepatic I/R-induced injury by inhibiting I/R-induced oxidative damage and liver cell apoptosis.
\end{abstract}

Correspondence to: Dr Jin Tao, Department of Nephrology, Xinxiang Central Hospital, 56 Jiansui Road, Xinxiang, Henan 453000, P.R. China

E-mail: xinxiangtaojin@163.com

Key words: tea polyphenols, ischemia/reperfusion, liver, oxidative damage, apoptosis

\section{Introduction}

Ischemia/reperfusion (I/R)-induced liver damage is a major complication following hemorrhagic shock, liver surgery and transplantation (1). Therefore, the development of effective strategies to treat hepatic I/R damage is important. Recently, a number of mechanisms have been shown to be involved in the process of I/R-induced injury in the liver, including oxidative stress and hepatic cell apoptosis $(2,3)$. These mechanisms are supported by research demonstrating that the inhibition of oxidative stress and hepatic cell apoptosis is effective in preventing I/R-induced injury in the liver $(4,5)$.

Tea polyphenols (TPs) are the primary active ingredients in green tea and are strong antioxidants that exert a significant free radical scavenging activity (6). A number of studies have demonstrated that TP can protect against oxidative stress in a number of organs, including the bones, liver and kidney (7-9); Yokozawa et al (9) reported that TP has a protective effect against renal damage caused by oxidative stress. It has also been demonstrated that TP can improve deficits in spatial cognitive ability resulting from cerebral hypoperfusion (10). Furthermore, TP has been observed to serve a protective role against apoptosis (11), and Xue et al (12) suggested that TP may attenuate neurocognitive impairment caused by global cerebral I/R injury via its anti-apoptotic properties. The role of $\mathrm{TP}$ in the protection of liver tissue against I/R-induced damage has been previously proposed. For instance, Zhong et al (13) demonstrated that green tea extract containing polyphenolic free radical scavengers prevented I/R-induced injury in the liver of rats. However, the specific mechanism remains uncertain.

In the present study, the mechanism underlying the protective effect of TPs against I/R-induced liver injury in mice was investigated, in particular focusing on its anti-oxidative and anti-apoptotic properties.

\section{Materials and methods}

Animals and ethical approval. The present study was approved by the Ethics Committee of Xinxiang Central Hospital (Xinxiang, China). Each experiment was performed in accordance with protocols set out by the Guidelines for the Care and Use of Experimental Animals (14). A total of 20 male C57BL/6 mice (Cavens Laboratory Animals Co., 
Ltd., (Changzhou, China), aged 12 weeks and weighing $25 \mathrm{~g}$, were used in the present study. Mice were housed in a laminar flow, temperature-controlled $\left(22 \pm 1^{\circ} \mathrm{C}\right)$, pathogen-free environment with a 12-h light/dark cycle and ad libitum access to food and water at the Experimental Animal Center of Xinxiang Medical School. Mice were fasted for $24 \mathrm{~h}$ prior to the experiments.

Pretreatment with TP. TP was purchased from Sigma-Aldrich (St. Louis, MO, USA) and was dissolved in saline according to the manufacturer's instructions. Mice were divided into four equal groups $(n=5)$ as follows: Saline-treated sham surgery mice (saline + sham); TP-treated sham surgery mice (TP + sham); saline-treated I/R injury mice (saline $+\mathrm{I} / \mathrm{R})$; and TP-treated I/R injury mice (TP + I/R). Saline or TP $(50 \mathrm{mg} / \mathrm{kg})$ was orally administered $1 \mathrm{~h}$ prior to surgery.

Induction of hepatic $I / R$ injury. An intraperitoneal injection of pentobarbital (50 mg/kg; Kehaojia Biological Technology, Wuhan, China) was used to anesthetize the animals. To induce I/R injury in the liver of the mice, a transverse incision was made to the abdomen and a micro clip (Hailunwentai, Shenzhen, China) was used to clamp the left branches of the portal vein and hepatic artery for $30 \mathrm{~min}$. Next, the clamp was removed and the wound was closed. In the sham surgery group, the same procedure was performed but the vessel was not occluded. The liver tissue and blood of mice were collected $6 \mathrm{~h}$ after the surgery.

Measurement of alanine aminotransferase (ALT) and aspartate aminotransferase (AST) serum activity. Blood was collected from the mice in each group. The Mouse Alanine Aminotransferase ELISA kit (MAK052) and the Mouse Aspartate Aminotransferase kit (MAK055; both Sigma-Aldrich) were used to determine the activity of serum ALT and AST, respectively, in accordance with the manufacturer's instructions.

Measurement of hepatic glutathione (GSH). Hepatic GSH and oxidized GSH (GSSG) levels were measured using a GSH and GSSG Assay kit (Beyotime Institute of Biotechnology, Shanghai, China). Following precipitation with $1 \%$ picric acid (Jinhao, Shanghai, China), the level of glutathione (GSH) was determined in liver homogenates using yeast-GSH reductase, 5,5'-Dithio-bis(2-nitrobenzoic acid) and NADPH (both Beyotime Institute of Biotechnology), and the absorbance was recorded at a wavelength of $412 \mathrm{~nm}$ using an ELx800 microplate reader (Biotek Instruments, Inc., Winooski, VT, USA), according to the manufacturer's protocol. The expression of GSSG in the presence of 2-vinylpyridine (Jinhao) was recorded using the same method. The ratio of GSH:GSSG was then calculated.

Flow cytometry. Flow cytometry was used to determine cell apoptosis using an Annexin-V-FITC Apoptosis Detection Kit I (BD Biosciences, Franklin Lake, NJ, USA). Briefly, hepatic cells were washed twice with cold PBS, and $10^{6}$ cells were subsequently resuspended in $200 \mu$ l binding buffer supplemented with $10 \mu \mathrm{l}$ Annexin-V-FITC and $5 \mu \mathrm{l}$ propidium iodide-phycoerythrin for incubation in the dark for $30 \mathrm{~min}$.
Following incubation, the cells were supplemented with $300 \mu \mathrm{l}$ binding buffer and analyzed using a C6 flow cytometer (BD Biosciences).

Reverse transcription-quantitative polymerase chain reaction $(R T-q P C R)$. Total RNA was extracted from liver tissue using TRIzol reagent (Thermo Fisher Scientific, Inc., Waltham, MA, USA) according to the manufacturer's instructions. RT and qPCR detection was performed using a SYBR Green RT PCR kit (Takara Bio, Inc., Otsu, Japan), according to the manufacturer's protocol. Reverse transcription was performed at $16^{\circ} \mathrm{C}$ for $30 \mathrm{~min}$, followed by an incubation step at $42^{\circ} \mathrm{C}$ for $30 \mathrm{~min}$ and enzyme inactivation at $85^{\circ} \mathrm{C}$ for $5 \mathrm{~min}$. Negative control (no cDNA) and RT control (no reverse transcription) were used. qPCR was performed to a final reaction volume of $20 \mu \mathrm{l}$ containing $0.5 \mu \mathrm{lcDNA}, 10 \mu \mathrm{lPCR}$ master mix (Takara Bio,Inc.), $2 \mu \mathrm{l}$ forward and reverse primers and $7.5 \mu \mathrm{l} \mathrm{H}_{2} \mathrm{O}$. PCR cycling conditions were as follows: $95^{\circ} \mathrm{C}$ for $5 \mathrm{~min}$, followed by 45 cycles of denaturation at $95^{\circ} \mathrm{C}$ for $15 \mathrm{sec}$ and annealing/elongation at $60^{\circ} \mathrm{C}$ for $30 \mathrm{sec}$. Specific primers were purchased from Sangon Biotech Co., Ltd., (Shanghai, China) as follows: Inducible nitric oxide synthase (iNOS) sense, 5'GTTCTCAGCCCA ACAATACAAGA'3, and anti-sense, 5'GTGGACGGGTCG ATGTCAC'3; B-cell lymphoma 2 (Bcl-2) sense, 5'ATGCCT TTGTGGAACTATATGGC'3, and anti-sense, 5'GGTATG CACCCAGAGTGATGC'3; Bcl-2-associated X protein (Bax) sense, 5'TGAAGACAGGGGCCTTTTTG'3 and anti-sense, 5'AATTCGCCGGAGACACTCG'3; and GAPDH sense, 5'AGGTCGGTGTGAACGGATTTG'3, and anti-sense, 5'TGT AGACCATGTAGTTGAGGTCA'3. GAPDH was used as an internal control. The relative expression of mRNA was quantified using GraphPad Prism version 4.0 (GraphPad Software, Inc., La Jolla, CA, USA) and the $2^{-\Delta \Delta \mathrm{Cq}} \operatorname{method}(15)$.

Western blot analysis. Total protein was extracted from the liver tissues in each group using a radioimmunoprecipitation assay solution (Sigma-Aldrich). The protein concentration was determined using a Bradford DC protein assay (Bio-Rad Laboratories, Inc., Hercules, CA, USA). To determine the expression level, protein $(20 \mu \mathrm{g})$ was separated using $10 \%$ sodium dodecyl sulfate-polyacrylamide gel electrophoresis, transferred to a polyvinylidene difluoride (PVDF) membrane (Thermo Fisher Scientific, Inc.) and incubated in Tris-buffered saline with Tween 20 (TBST; Sigma-Aldrich) and $50 \mathrm{~g} / 1$ skimmed milk at room temperature for $3 \mathrm{~h}$. The PVDF membrane was then incubated at room temperature for $3 \mathrm{~h}$ with the following rabbit monoclonal primary antibodies (all from Abcam, Cambridge, MA, USA): Anti-iNOS (1:100; ab15323), anti-Bax (1:50; ab32503), anti-cytochrome $c$ (1:100; ab133504), anti-Bcl-2 (1:200; ab32124) or anti-GAPDH (1:200; ab8245). Next, the PVDF membranes were washed with TBST three times and then incubated with a mouse anti-rabbit secondary antibody (1:20,000; ab99697; Abcam) at room temperature for $1 \mathrm{~h}$. An enhanced chemiluminescence kit (Pierce Biotechnology, Rockford, IL, USA) was used to perform chemiluminescent detection. Results were quantified using ImageJ software (National Institutes of Health, Bethesda, MA, USA).

Measurement of caspase-3 activity. The activity of caspase-3 was determined using a Caspase-3 Colorimetric 
Assay kit (BioVision, Inc., Milpitas, CA, USA), according to the manufacturer's instructions. Protein $(20 \mu \mathrm{g})$ from liver tissues was incubated in the solution buffer provided with the kit at room temperature for $30 \mathrm{~min}$ Next, $200 \mu \mathrm{M}$ N-acetyl-Asp-Glu-Val-Asp-7-amino(4-trifluoromethyl)-coumarin was added and the samples were incubated at $37^{\circ} \mathrm{C}$ for $2 \mathrm{~h}$. The absorbance was measured spectrophotometrically at $400 \mathrm{~nm}$ using an ELx800 microplate reader.

Statistical analysis. The mean \pm standard error of the data was calculated and analyzed using one-way analysis of variance. SPSS version 17.0 (SPSS, Inc., Chicago, IL, USA) was used to perform statistical analyses. $\mathrm{P}<0.05$ was considered to indicate a statistically significant difference.

\section{Results}

Pretreatment with TP attenuates the upregulation of serum ALT and AST activity in mice with I/R-induced liver injury. To evaluate the extent of hepatic injury in mice, the activity of serum ALT and AST was measured in each group. As presented in Fig. 1A, the serum activity of ALT was significantly upregulated in the saline $+\mathrm{I} / \mathrm{R}$ group in comparison with the saline + sham group $(\mathrm{P}<0.01)$, suggesting that the liver was damaged by the I/R-induced injury. However, this increase was significantly attenuated by pre-treatment with $\mathrm{TP}$ $(\mathrm{P}<0.01$; Fig. 1A). There was no significant difference in the serum activity of ALT between the saline + sham group and the $\mathrm{TP}+$ sham group.

Similar results were observed in the activity of serum AST. As presented in Fig. 1B, the serum activity of AST was significantly increased in the saline $+I / R$ group in comparison with the saline + sham group $(\mathrm{P}<0.01)$, which was markedly attenuated by pre-treatment with $\mathrm{TP}(\mathrm{P}<0.01)$. By contrast, no significant difference in the serum activity of AST was observed between the saline + sham and TP + sham groups. Based on these observations, it can be suggested that pretreatment with TP attenuates I/R-induced liver injury in mice.

Pretreatment with TP attenuates the decease in GSH/GSSG ratio in mice with $I / R$-induced liver injury. The content of GSH in the liver of mice was observed in each group. As presented in Fig. 2, the GSH/GSSG ratio in the saline + I/R group was significantly decreased in comparison with the saline + sham group $(\mathrm{P}<0.01)$, suggesting that the liver was injured by I/R. However, this downregulation was attenuated in the $\mathrm{TP}+\mathrm{I} / \mathrm{R}$ group in comparison with the saline $+\mathrm{I} / \mathrm{R}$ group $(\mathrm{P}<0.01$; Fig. 2$)$, suggesting that pretreatment with $\mathrm{TP}$ attenuated the I/R-induced liver injury in mice. However, no statistically significant difference in the GSH/GSSG ratio was detected between the saline + sham group and the $\mathrm{TP}+$ sham group.

Pretreatment with TP suppresses the downregulation of iNOS in mice with I/R-induced liver injury. iNOS is known to participate in a host's defense against oxidative damage (16); therefore, the mRNA and protein expression of iNOS in each group was analyzed. As demonstrated in Fig. 3A and B, the
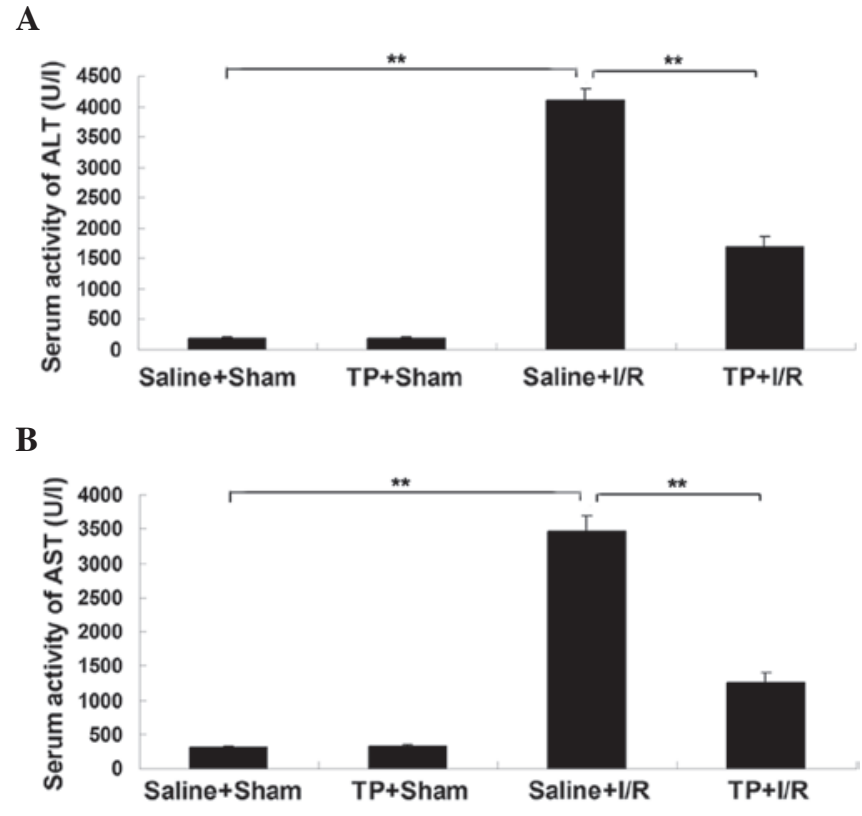

Figure 1. Serum activity of (A) ALT and (B) AST in blood. ${ }^{* *} \mathrm{P}<0.01$ $\mathrm{I} / \mathrm{R}$, ischemia/reperfusion; TP, tea polyphenols; ALT, alanine aminotransferase; AST, aspartate aminotransferase.

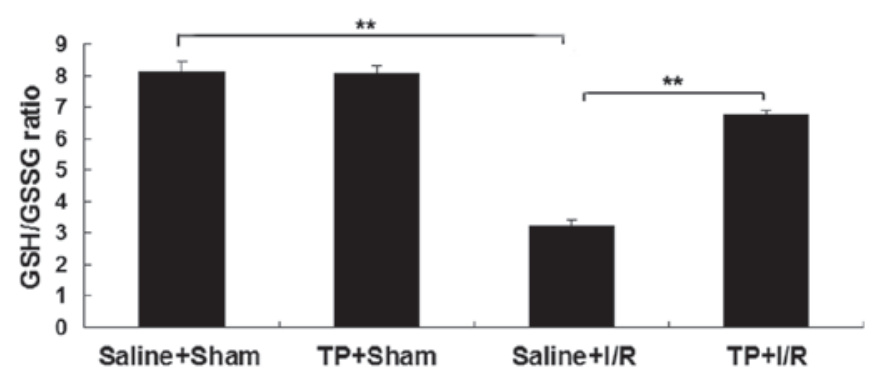

Figure 2. Ratio of GSH/GSSG in liver homogenates. ${ }^{* *} \mathrm{P}<0.01$. I/R, ischemia/reperfusion; TP, tea polyphenols; GSH, glutathione; GSSG, oxidised glutathione.

mRNA and protein expression levels of iNOS were significantly decreased in the saline $+\mathrm{I} / \mathrm{R}$ group in comparison with the saline + sham group $(\mathrm{P}<0.01)$; however, this downregulation was significantly attenuated by pretreatment with TP $(\mathrm{P}<0.01)$. Furthermore, there was no significant difference in the iNOS levels between the saline + sham group and $\mathrm{TP}+$ sham groups.

Pretreatment with TP attenuates I/R-induced hepatic cell apoptosis in mice. The level of apoptosis in liver tissues in each group was analyzed. As presented in Fig. 4, the level of apoptosis was significantly upregulated in the liver tissues of mice in the saline $+\mathrm{I} / \mathrm{R}$ group in comparison with the saline + sham group $(\mathrm{P}<0.01)$, indicating that $\mathrm{I} / \mathrm{R}$ injury induced cell apoptosis in the liver of mice. However, the level of apoptosis was significantly reduced in the $\mathrm{TP}+\mathrm{I} / \mathrm{R}$ group in comparison with the saline $+I / R$ group $(\mathrm{P}<0.01$; Fig. 4$)$, suggesting that pretreatment with TP attenuated I/R-induced hepatic cell apoptosis in mice. However, there was no significant difference in the level of apoptosis between the saline + sham and $\mathrm{TP}+$ sham groups. 
A

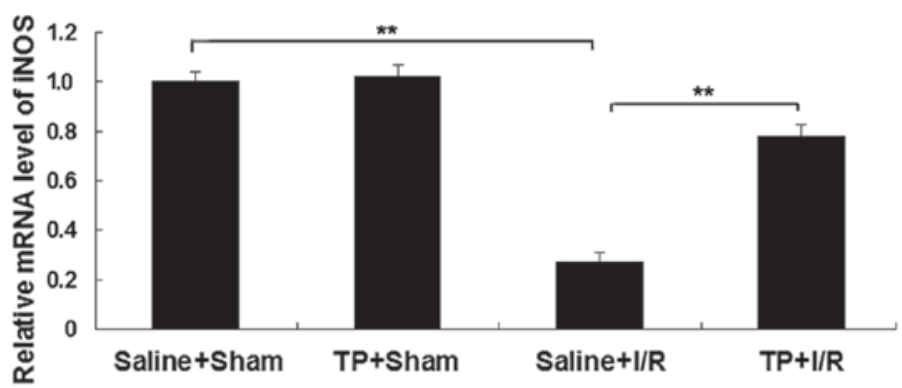

B

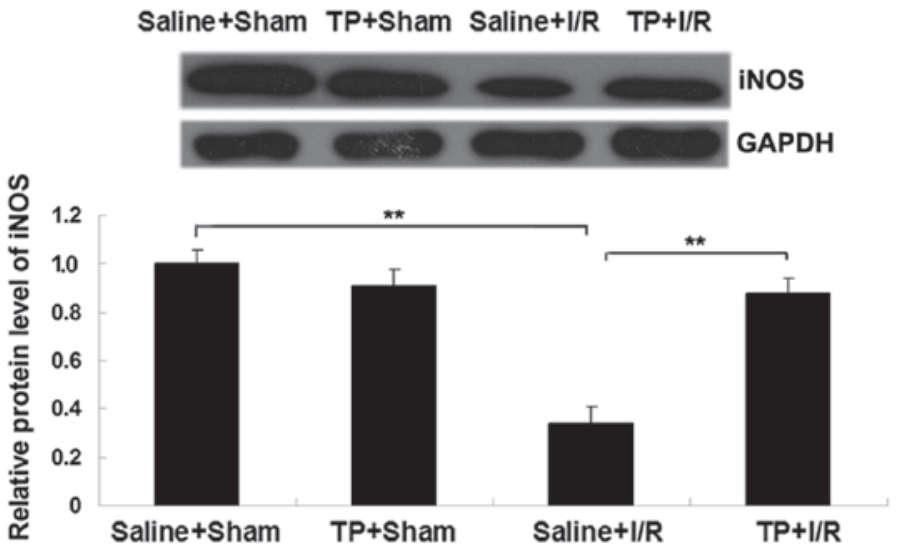

Figure 3. Levels of iNOS (A) mRNA and (B) protein. GAPDH was used as an internal control. ${ }^{* *} \mathrm{P}<0.01$. I/R, ischemia/reperfusion; TP, tea polyphenols; iNOS, cytokine-inducible nitric oxide synthase.

A



TP+Sham

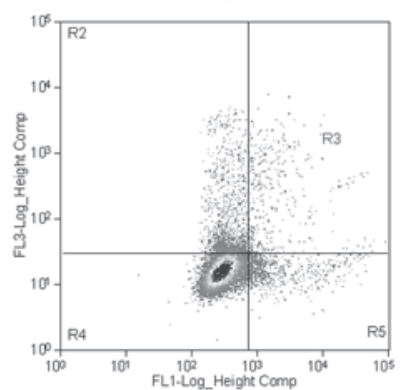

Saline+1/R

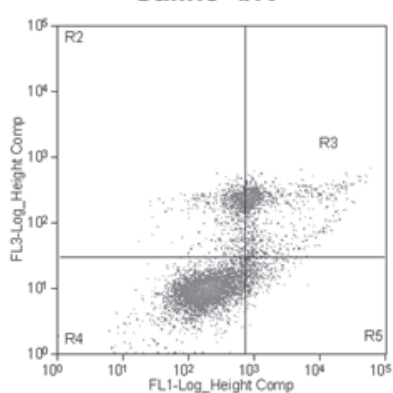

$\mathrm{TP}+\mathrm{I} / \mathrm{R}$

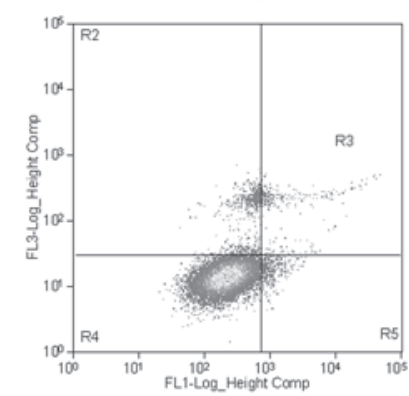

B

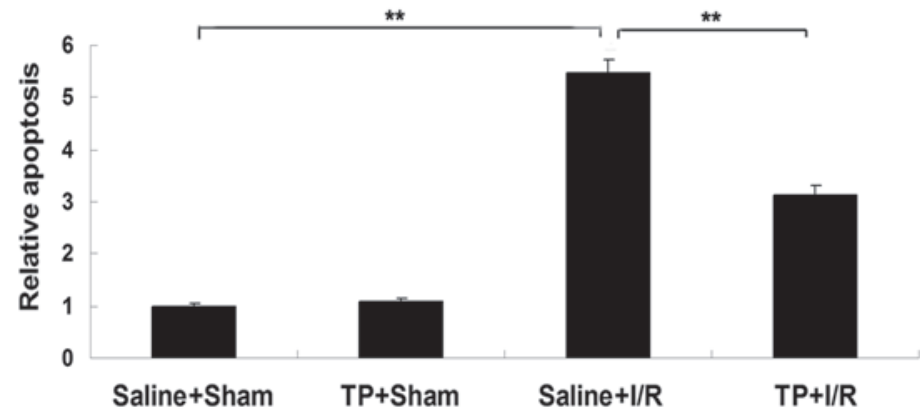

Figure 4. (A) Scatter plot and (B) quantification of apoptosis. ${ }^{* *} \mathrm{P}<0.01$. FL1, Annexin V-fluorescein isothiocyanate; FL3, propidium iodide; I/R, ischemia/reperfusion; TP, tea polyphenols.

The mRNA and protein expression levels of two key apoptosis-associated proteins, Bcl-2 and Bax, were also analyzed in each group. As demonstrated in Fig. 5A and B, the mRNA and protein levels of pro-apoptotic Bax were significantly increased in the saline $+\mathrm{I} / \mathrm{R}$ group in comparison with the saline + sham group $(\mathrm{P}<0.01)$. However, this upregulation was markedly attenuated by the pretreatment with $\mathrm{TP}(\mathrm{P}<0.01$; Fig. 5A and B). Although the mRNA and protein expression 
A

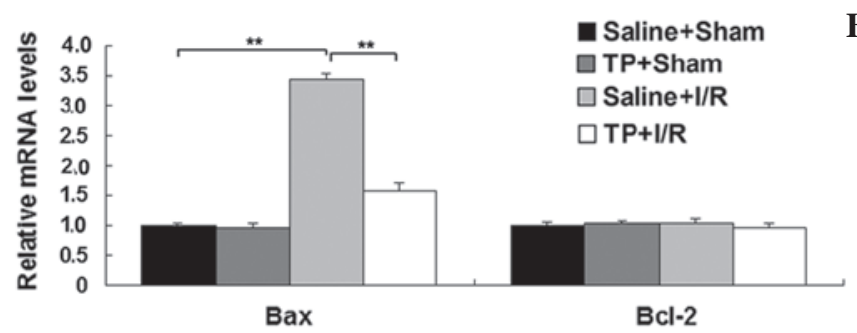

C



$\mathbf{E}$

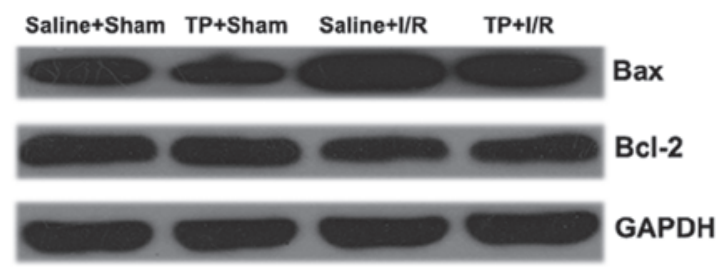

B

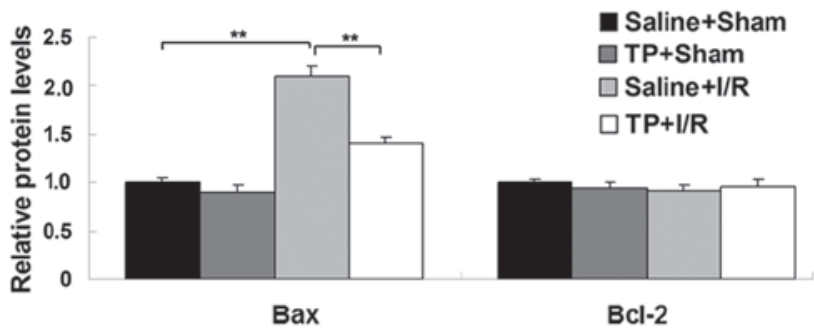

D
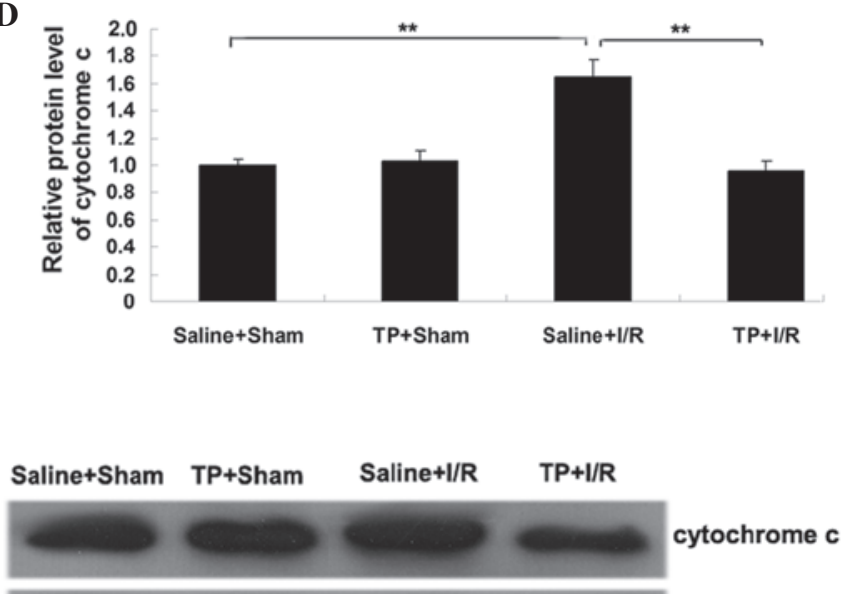

GAPDH

Figure 5. Relative (A) mRNA and (B) protein expression levels of Bax and Bcl-2. (C) Relative Bax/Bcl-2 ratio. (D) Relative protein expression level of cytochrome $c$ in cytosol. (E) Western blots of Bax, Bcl-2 and cytochrome $c$ protein expression levels, with GAPDH used as an internal control. ${ }^{* *} \mathrm{P}<0.01$. $\mathrm{I} / \mathrm{R}$, ischemia/reperfusion; TP, tea polyphenols; Bcl-2, B-cell lymphoma 2; Bax, Bcl2-associated X protein.

A

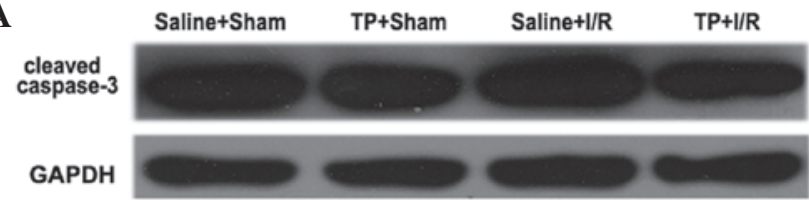

B

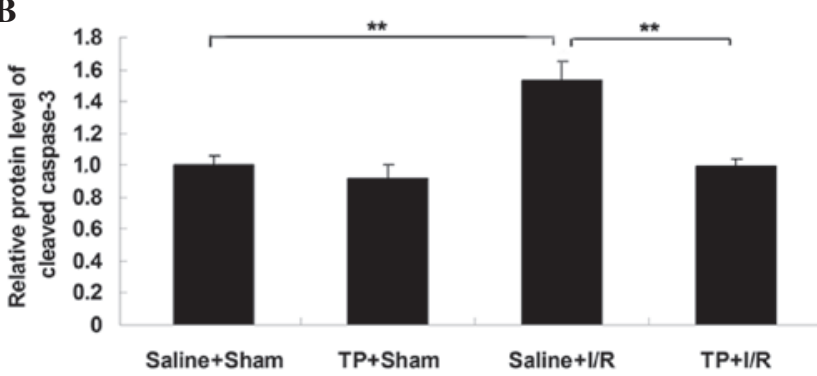

C

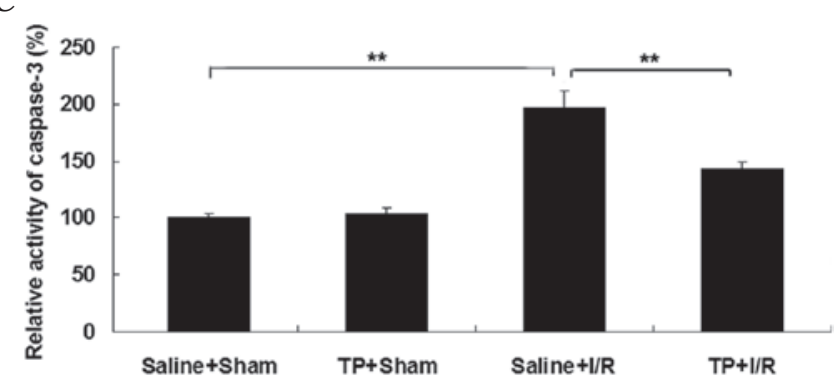

Figure 6. (A) Western blot analysis of caspase-3 expression levels. GAPDH was used as an internal control. (B) Relative protein expression of cleaved caspase-3 and (C) relative activity of caspase-3. ${ }^{* *} \mathrm{P}<0.01 . \mathrm{I} / \mathrm{R}$, ischemia/reperfusion; TP, tea polyphenols. levels of Bcl-2 were not significantly different between groups (Fig. 5A and B), the Bax/Bcl-2 ratio was significantly increased in the saline $+\mathrm{I} / \mathrm{R}$ group in comparison with that in the saline + sham group $(\mathrm{P}<0.01$; Fig. $5 \mathrm{C})$, and was significantly attenuated by pretreatment with TP $(\mathrm{P}<0.01)$. Similar findings were observed in the protein expression levels of cytochrome $c$. In addition, cytochrome $c$ expression levels were significantly increased in the saline $+I / R$ group in comparison with those in the saline + sham group $(\mathrm{P}<0.01$; Fig. 5D), which was significantly attenuated by pretreatment with TP $(\mathrm{P}<0.01)$.

Furthermore, the expression levels and activity of caspase- 3 in the liver were analyzed in each group. As presented in Fig. 6A and B, the protein expression level of cleaved caspase- 3 was significantly upregulated in the saline $+I / R$ group compared with those in the saline + sham group $(\mathrm{P}<0.01)$, suggesting that the activation of caspase- 3 is involved in I/R-induced liver cell apoptosis. However, pretreatment with TP significantly attenuated I/R-induced upregulation of cleaved caspase-3 expression in liver tissues in mice $(\mathrm{P}<0.01$; Fig. $6 \mathrm{~A}$ and $\mathrm{B})$. Consistent with these data, the activity of caspase-3 was also significantly upregulated in the saline $+I / R$ group compared with the saline + sham group $(\mathrm{P}<0.01)$, which was also significantly attenuated by pretreatment with TP $(\mathrm{P}<0.01)$. Taken together these findings suggested that TP may at least partially protect against hepatic I/R injury in mice, through the inhibition of the expression of cytokine inducible nitric oxide synthase in liver tissues, and the apoptosis of liver cells. 


\section{Discussion}

TPs have been shown to protect against carbon tetrachloride and lipopolysaccharide-induced liver injury, hepatic I/R injury and liver fibrosis $(13,17-19)$. However, the precise mechanism underlying TPs protective effect against I/R-induced liver injury remains uncertain. In the present study, it was demonstrated that TP protected against I/R-induced liver injury in mice using anti-oxidative and anti-apoptotic properties.

The activities of serum ALT and AST are important indicators of oxidative damage in liver tissues $(20,21)$. In the current study, it was demonstrated that the upregulation of ALT and AST activity following I/R injury was attenuated by pretreatment with TP in mice. Furthermore, GSH serves as an antioxidant in removing reactive oxygen in human tissue, while GSSG can be converted to GSH by glutathione reductase (22). In the present study, it was observed that the administration of TP effectively attenuated the I/R-induced decrease in the GSH/GSSG ratio.

It has been reported that overexpression of iNOS can protect against hepatic I/R injury by modulating oxidative stress (23). Therefore, the mRNA and protein expression levels of iNOS were investigated in the different groups of the current study. The results demonstrated that pretreatment with $\mathrm{TP}$ attenuated the downregulation of iNOS in I/R-induced injured liver tissues in mice. Based on these results, it can be suggested that TP protects against I/R-induced liver injury by inhibiting oxidative damage. In addition, I/R can induce cell apoptosis in a number of organs including the liver, and the inhibition of cell apoptosis effectively attenuates I/R-induced liver damage $(24,25)$. The present study revealed that pretreatment with TP attenuated I/R-induced liver cell apoptosis.

A number of proteins, including Bax, $\mathrm{Bcl}-2$, cytochrome $c$ and caspase-3, have been demonstrated to serve key roles in I/R-induced cell apoptosis. Bax is an important member of the Bcl-2 family and can promote cell apoptosis via a mitochondrial-mediated apoptosis pathway (26). Bcl-2 inhibits cell endoplasmic reticulum $\mathrm{Ca}^{2+}$ release, lipid peroxide formation and free radical production, and thus plays a suppressive role in cell apoptosis (27). However, Bax can bind to Bcl-2 and inhibit its anti-apoptotic activity (28). In the present study, although the expression level of Bcl-2 presented no significant changes following I/R-induced injury, Bax expression levels and the $\mathrm{Bax} / \mathrm{Bcl}-2$ ratio were significantly upregulated in I/R-induced injured liver tissues, which was significantly attenuated by pretreatment with TP.

When cell apoptosis occurs, the mitochondrial membrane potential collapses and cytochrome $c$ moves into the cytosol (29); therefore, the expression level of cytochrome $c$ was analyzed in the cytosol of each group in the present study. The results demonstrated that cytochrome $c$ expression was significantly upregulated in I/R-induced liver tissues, which was significantly attenuated by pretreatment with TP.

Caspase-3 is a key member of the cysteine-aspartate-specific protease family, and has been demonstrated to function as an ultimate enforcer during cell apoptosis. Upregulated expression, in addition to increased activation, of caspase- 3 has been reported in apoptotic cells $(30,31)$. In addition, the upregulation of caspase- 3 has been reported in I/R-induced hepatic injury, indicating that caspase-mediated cell apoptosis serves a crucial role in $\mathrm{I} / \mathrm{R}$-induced organ damage (32). In the present study, the expression level and activity of caspase- 3 was examined in each group and it was observed that pretreatment with TP significantly attenuated the expression and activity of caspase-3 in I/R injured liver tissues in mice. Based on these observations, it can be suggested that TP can attenuate I/R-induced liver cell apoptosis by inhibiting the upregulation of Bax, caspase-3, and the release of cytochrome $c$. Therefore, the results of the present study demonstrated that $\mathrm{TP}$ has a protective effect against hepatic I/R injury in mice via its anti-oxidative function, which inhibits the expression of cytokine inducible nitric oxide synthase in liver tissues. Furthermore, TP was also capable of inhibiting the I/R-induced apoptosis of liver cells via the downregulation of pro-apoptotic $\mathrm{BAX}$ and upregulation of anti-apoptotic Bcl2.

In conclusion, the present study demonstrated that oral administration of TP can attenuate I/R-induced hepatic injury via the inhibition of oxidative damage and liver cell apoptosis. Therefore, TP may be a potential candidate for treating hepatic injury.

\section{References}

1. Mendes-Braz M, Elias-Miró M, Jiménez-Castro MB, Casillas-Ramírez A, Ramalho FS and Peralta C: The current state of knowledge of hepatic ischemia-reperfusion injury based on its study in experimental models. J Biomed Biotechnol 2012: 298657, 2012.

2. Zhuonan Z, Sen G, Zhipeng J, Maoyou Z, Linglan Y, Gangping W, Cheng J, Zhongliang M, Tian J, Peijian Z and Kesen X: Hypoxia preconditioning induced HIF-1 $\alpha$ promotes glucose metabolism and protects mitochondria in liver I/R injury. Clin Res Hepatol Gastroenterol 39: 610-619, 2015.

3. Trocha M, Merwid-Ląd A, Chlebda E, Sozański T, Pieśniewska M, Gliniak $\mathrm{H}$ and Szeląg A: Influence of ezetimibe on selected parameters of oxidative stress in rat liver subjected to ischemia/reperfusion. Arch Med Sci 10: 817-824, 2014.

4. Bae UJ, Yang JD, Ka SO, Koo JH, Woo SJ, Lee YR, Yu HC, Cho BH, Zhao HY, Ryu JH, et al: SPA0355 attenuates ischemia/reperfusion-induced liver injury in mice. Exp Mol Med 46: e109, 2014.

5. Sheng M, Zhou Y, Yu W, Weng Y, Xu R and Du H: Protective effect of Berberine pretreatment in hepatic ischemia/reperfusion injury of rat. Transplant Proc 47: 275-282, 2015.

6. Rehman H, Krishnasamy Y, Haque K, Thurman RG, Lemasters JJ, Schnellmann RG and Zhong Z: Green tea polyphenols stimulate mitochondrial biogenesis and improve renal function after chronic cyclosporin a treatment in rats. PLoS One 8: e65029, 2014

7. Shen CL, Chyu MC and Wang JS: Tea and bone health: Steps forward in translational nutrition. Am J Clin Nutr 98 (Suppl 6): 1694S-1699S, 2013.

8. Clifford MN, van der Hooft JJ and Crozier A: Human studies on the absorption, distribution, metabolism, and excretion of tea polyphenols. Am J Clin Nutr 98 (Suppl 6): 1619S-1630S, 2013.

9. Yokozawa T, Noh JS and Park CH: Green tea polyphenols for the protection against renal damage caused by oxidative stress. Evid Based Complement Alternat Med 2012: 845917, 2012.

10. Xu Y, Zhang JJ, Xiong L, Zhang L, Sun D and Liu H: Green tea polyphenols inhibit cognitive impairment induced by chronic cerebral hypoperfusion via modulating oxidative stress. J Nutr Biochem 21: 741-748, 2010.

11. Wang M and Lei YX: Effects of tea polyphenols on proliferation and apoptosis of cadmium-transformed cells. Int J Clin Exp Med 8: 3054-3062, 2015.

12. Xue R, Wu G, Wei X, Lv J, Fu R, Lei X, Zhang Z, Li W, He J, et al: Tea polyphenols may attenuate the neurocognitive impairment caused by global cerebral ischemia/reperfusion injury via anti-apoptosis. Nutr Neurosci: Nov 20, 2014 (Epub ahead of print).

13. Zhong Z, Froh M, Connor HD, Li X, Conzelmann LO, Mason RP, Lemasters JJ and Thurman RG: Prevention of hepatic ischemia-reperfusion injury by green tea extract. Am J Physiol Gastrointest Liver Physiol 283: G957-G964, 2002. 
14. Institute of Laboratory Animal Resources (US). Committee on Care, Use of Laboratory Animals, and National Institutes of Health (US). Division of Research Resources: Guide for the care and use of laboratory animals. 8th edition. National Academies Press, Washington, DC, 2011

15. Mahale A, Othman MW, Al Shahwan S, Al Jadaan I, Owaydha O, Khan Z and Edward DP: Altered expression of fibrosis genes in capsules of failed Ahmed glaucoma valve implants. PLoS One 10 e0122409, 2015

16. Li YN, Wang XJ, Li B, Liu K, Qi JS, Liu BH and Tian Y: Tongxinluo inhibits cyclooxygenase-2, inducible nitric oxide synthase, hypoxia-inducible factor- $2 \alpha$ /vascular endothelial growth factor to antagonize injury in hypoxia-stimulated cardiac microvascular endothelial cells. Chin Med J (Engl) 128: 1114-1120, 2015.

17. Cui Y, Yang X, Lu X, Chen J and Zhao Y: Protective effects of polyphenols-enriched extract from Huangshan Maofeng green tea against $\mathrm{CCl}_{4}$-induced liver injury in mice. Chem Biol Interact 220 $75-83,2014$.

18. Yuan GJ, Gong ZJ, Sun XM, Zheng SH and Li X: Tea polyphenols inhibit expressions of iNOS and TNF-alpha and prevent lipopolysaccharide-induced liver injury in rats. Hepatobiliary Pancreat Dis Int 5: 262-267, 2006.

19. Li YM, Zhang XG, Zhou HL, Chen SH, Zhang Y and $\mathrm{Yu} \mathrm{CH}$ Effects of tea polyphenols on hepatic fibrosis in rats with alcoholic liver disease. Hepatobiliary Pancreat Dis Int 3: 577-579, 2004

20. Zhong $\mathrm{Z}$ and Lemasters JJ: Role of free radicals in failure of fatty liver grafts caused by ethanol. Alcohol 34: 49-58, 2004.

21. Cetinkunar S, Tokgoz S, Bilgin BC, Erdem H, Aktimur R Can S, Erol HS, Isgoren A, Sozen S and Polat Y: The effect of silymarin on hepatic regeneration after partial hepatectomy: Is silymarin effective in hepatic regeneration? Int J Clin Exp Med 8: 2578-2585, 2015.

22. Korge P, Calmettes G and Weiss JN: Increased reactive oxygen species production during reductive stress: The roles of mitochondrial glutathione and thioredoxin reductases. Biochim Biophys Acta 1847: 514-525, 2015.
23. Tao X, Wan X, Xu Y, Xu L, Qi Y, Yin L, Han X, Lin Y and Peng J: Dioscin attenuates hepatic ischemia-reperfusion injury in rats through inhibition of oxidative-nitrative stress, inflammation and apoptosis. Transplantation 98: 604-611, 2014.

24. Guo Y, Hu B, Huang H, Tsung A, Gaikwad NW, Xu M, Jiang M, Ren S, Fan J, Billiar TR, et al: Estrogen sulfotransferase is an oxidative stress responsive gene that gender-specifically affects liver ischemia/reperfusion injury. J Biol Chem 290: 14754-14764, 2015.

25. Yan Y, Li G, Tian X, Ye Y, Gao Z, Yao J, Zhang F and Wang S: Ischemic preconditioning increases GSK-3 $/ \beta$-catenin levels and ameliorates liver ischemia/reperfusion injury in rats. Int $\mathrm{J}$ Mol Med 35: 1625-1632, 2015

26. Renault TT, Teijido O, Antonsson B, Dejean LM and Manon S: Regulation of Bax mitochondrial localization by Bcl-2 and $\mathrm{Bcl}-\mathrm{x}(\mathrm{L})$ : Keep your friends close but your enemies closer. Int J Biochem Cell Biol 45: 64-67, 2013.

27. Shamas-Din A, Kale J, Leber B and Andrews DW: Mechanisms of action of Bcl-2 family proteins. Cold Spring Harb Perspect Biol 5: a008714, 2013.

28. Renault TT and Manon S: Bax: Addressed to kill. Biochimie 93: 1379-1391, 2011.

29. Bernardi P and Rasola A: Calcium and cell death: The mitochondrial connection. Subcell Biochem 45: 481-506, 2007.

30. McIlwain DR, Berger T and Mak TW: Caspase functions in cell death and disease. Cold Spring Harb Perspect Biol 5: a008656, 2013.

31. Hua P, Liu J, Tao J, Liu J and Yang S: Influence of caspase-3 silencing on the proliferation and apoptosis of rat bone marrow mesenchymal stem cells under hypoxia. Int J Clin Exp Med 8: 1624-1633, 2015.

32. Qin Y, Vanden Hoek TL, Wojcik K, Anderson T, Li CQ, Shao ZH, Becker LB and Hamann KJ: Caspase-dependent cytochrome c release and cell death in chick cardiomyocytes after simulated ischemia-reperfusion. Am J Physiol Heart Circ Physiol 286: H2280-H2286, 2004. 